\title{
MODELAGEM MATEMÁTICA DO PROCESSO DE EXTRAÇÃO DE ÓLEOS ESSENCIAIS Schinus terebintifolius Raddi UTILIZANDO FLUIDOS SUPERCRÍTICOS
}

Ana Carolina Araújo dos Santos ${ }^{1}$; Gabriel Antônio Batista Nascimento2; Natalia Barbosa ${ }^{3}$; Victor Laurent ${ }^{4}$; Yan Valdez Santos Rodrigues5; Ana Lúcia Barbosa6; Ewerton Emmanuel da Silva Calixto ${ }^{7}$, Fernando Luiz Pellegrini Pessoa ${ }^{8}$

${ }^{1}$ Centro Universitário SENAI CIMATEC; Salvador-BA; anacarolina28160@gmail.com 2,3,4,5,6,7,8 Centro Universitário SENAI CIMATEC; Salvador-BA;

Resumo: Schinus terebinthifolius Raddi é uma planta rica em nutrientes e é utilizada tanto de forma medicinal como na área industrial. $O$ interesse comercial na extração do óleo da S. terebinthifolius, faz com que métodos de extração mais baratos como o supercrítico sejam vistos como promissores na indústria na atualidade. Os modelos matemáticos como Sovová (1994) e Esquivel (1999) são utilizados para descrever o comportamento de extrações supercriticas. Nesse trabalho, o objetivo foi comparar esses modelos e dados experimentais para pressão 223 bar e a temperatura $50^{\circ} \mathrm{C}$, e verificar rendimentos e qual modelo mais adequado. $E$ a partir do estudo, observouse bons rendimentos para essa pressao e temperatura e verificou-se que o Sovová (1994) proporcionou boa reprodutibilidade e representatividade.

Palavras-Chave: Schinus terebinthifolius Raddi; extração supercrítica; Esquivel; Sovová.

\section{MATHEMATICAL MODELING OF THE ESSENTIAL OIL EXTRACTION PROCESS OF Schinus terebintifolius Raddi Using SUPERCRITIC FLUIDS}

Abstract: Schinus terebinthifolius Raddi is a plant rich in nutrients and is used both medicinally and industrially. The commercial interest in S. terebinthifolius oil extraction makes cheaper extraction methods such as supercritical to be seen as promising in the industry today. Mathematical models such as Sovová (1994) and Esquivel (1999) are used to describe the behavior of supercritical extractions. In this work, the objective was to compare these models and experimental data for pressure 223 bar and temperature $50^{\circ} \mathrm{C}$, and to verify yields and which model is most appropriate. And from the study, it was observed good yields for this pressure and temperature and it was found that Sovová (1994) provided good reproducibility and representativeness.

Keywords: Schinus terebinthifolius Raddi; supercritical extraction; Esquivel; Sovová. 


\section{INTRODUÇÃO}

O Brasil possui, em abundância, espécies vegetais com grande potencial econômico, de uso medicinal, rural e também industrial, a aroeira, Schinus terebinthifolius Raddi, é uma planta rica em nutrientes e utilizada tanto de forma medicinal como na área industrial por possuir componentes químicos e carotenoides.

Existem vários métodos de extrações que podem ser utilizados atualmente, porém um dos métodos mais promissores para indústria é a extração supercrítica, pelo fato dela não deixar nenhum traço do solvente no produto, de ter uma excelente qualidade e pelo próprio fato da legislação ambiental proibir resíduos de solventes em processos industriais. Segundo Brunner, nenhuma substância é um fluido supercrítico, mas pode ser levado a esse estado elevando seu calor e pressão até supera seu ponto crítico, portanto um fluido supercrítico é um fluido puro o qual acima de tais características. O solvente mais utilizado nesse tipo de extração é o $\mathrm{CO}_{2}$, por ser o mais seguro, baixo custo e como também os outros fluidos geralmente serem caros, de baixo acesso ou até mesmo deixarem resíduos após a extração.

O modelo de Sovová considera que o material a ser extraído contém células intactas no seu interior e as células externas estão rompidas envoltos em uma estufa de formato esférico, Sovová também considerou que o solvente e o soluto estão em equilíbrio no interior da célula durante a pressurização com a concentração inalterada no a célula intacta. [3]

Esquível propôs um modelo com o intuito de expor as curvas de extração de fácil modelagem usando uma equação empírica para o ajuste da curva experimental, sendo esse modelo empírico, o mesmo não considera interações entre o soluto e a matriz [4]. Esse artigo tem como objetivo obter um modelo matemático apropriado das curvas de extração: produção de óleo essencial versus tempo de extração, a partir dos dados experimentais.

\section{METODOLOGIA}

\subsection{Dados e Ferramentas usados}

Para a composição do trabalho foi utilizado dados providos pela professora Ana Lúcia que são referentes ao processo de extração nas temperaturas de $50^{\circ} \mathrm{C}$ e pressão de 223 bar para construção de tabelas, cálculos e gráficos usou-se o Excel@ e seu suplementos "Solver".

\subsection{Curvas de Extração}

Uma curva de extração é constituída de três etapas, como apresentado na Figura 1: - $\quad$ Etapa de taxa de extração constante (CER) - predomínio da transferência de massa devido à convecção na fase fluida;

- $\quad$ Etapa de taxa de extração decrescente (FER) - efeitos convectivos na fase fluida e difusionais na fase sólida determinam a velocidade do processo;

- $\quad$ Etapa de taxa de extração quase nula (DC) - predomínio do efeito difusional. 


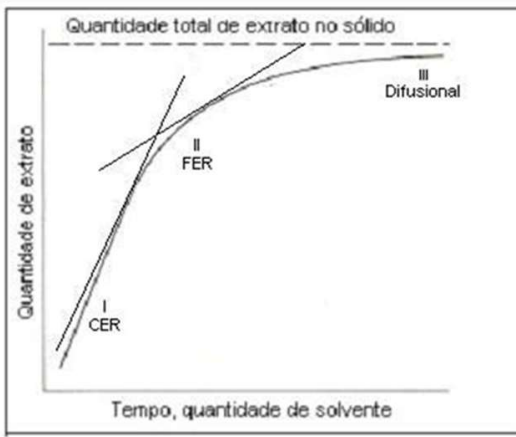

Figura 1: Curva de extração (ALMEIDA, 2006), com duas retas ajustadas.

Cada etapa foi caracterizada pelo domínio de um fenômeno ou mais de um característico da transferência de massa, dessa forma a parte inicial da curva representa uma reta que indica uma taxa de extração constante, ou seja, caracterizada pela transferência de massa por convecção entre a superfície do sólido e o solvente. Após, segue-se uma etapa de transição, durante a qual a taxa de extração cai rapidamente logo uma mudança do domínio da convecção para difusão, assim, o processo difusional começa a controlar a transferência de massa e a inclinação da curva diminui e torna-se assintótica [1].

\subsection{Modelo de Sovová (1994)}

Esse modelo considera um estado pseudo-estacionário, em que a temperatura, a pressão e a velocidade do solvente são mantidas constantes, há escoamento axial do solvente com velocidade superficial através de um leito fixo de seção transversal cilíndrica e que o soluto está contido no interior das células nas plantas. Também hipotetiza-se que o pré-tratamento do material, como uma trituração, que aumenta a superfície de contato entre o sólido e o solvente, separar a quantidade total de soluto disponível em duas partes sendo uma de fácil e outra de difícil acesso. [3]

Em que cada fase da curva de extração foi determinada pelas equaçoes na Figura 2 abaixo:

Figura 2: Equações referentes as fases da curva de extração. Fonte: (ALMEIDA, 2006)

$$
\begin{gathered}
C E R=\left(\frac{X_{K} \tau}{Z}\right) *\left(1-e^{(-Z)}\right) \text { para } \tau<\tau_{m} \\
F E R=\left(\frac{X_{K}}{Z}\right) *\left(\tau-\tau_{m} * e^{\left(Z_{w}-Z\right)}\right) \text { para } \tau_{m} \leq \tau<\tau_{n}(2) \\
D C=X_{0}-\left(\frac{X_{K}}{K Z}\right) * \ln \left(1+e^{\left(r_{0} * K * Z\right)}-1\right) * \frac{e^{\left(K *\left(\tau_{m}-\tau\right)\right)}}{r_{0}} \text { para } \tau>\tau_{n}
\end{gathered}
$$

$\tau$ é o criterio para definir a fase dominante em deteriminado intervalo de tempo do processo de extração e podem ser calculados atraves:

$$
\begin{gathered}
\tau=\frac{k_{f} a_{o} \times \rho \times y_{r}}{(1-\varepsilon) \times \rho_{s} \times X_{k}} \times t \\
\tau_{n}=\tau_{m}+\frac{1}{K} \times \ln \left(\frac{1+\tau_{m} \exp \left(r_{o} \times K \times Z\right)}{1+\tau_{m}}\right)
\end{gathered}
$$




$$
\tau_{m}=r_{o}-1
$$

È os parametros $\mathrm{kf}_{\mathrm{f}} \mathrm{yr}_{\mathrm{r}} \mathrm{r}, \mathrm{K}, \mathrm{Z}, \mathrm{Zw}, \mathrm{U}, \mathrm{q}, \mathrm{M}_{\mathrm{sf}}$ e outros, são dados por:

$$
\begin{aligned}
& y_{r}=\left[\exp \left(40,361-\frac{18708}{T}+\frac{2186840}{T^{2}}\right) \times(0,001 \rho)^{10,724} \pm 2,7\right] / \rho \\
& Z=\frac{k_{f} a_{o} \times \rho}{\left[q \times(1-\varepsilon) \times \rho_{s}\right]} \\
& r_{o}=X_{o} / X_{k} \\
& K=\frac{k_{s} \times \rho_{s} \times X_{k}}{k_{f} \times \rho \times y_{r}} \\
& U=Q_{\mathrm{CO}_{2}} \times A_{c} \\
& Z w=\frac{1}{K \times r_{o}} \times \ln \left(\frac{r_{o} \times \exp \left(K \times\left(\tau-\tau_{m}\right)\right)-1}{r_{o}-1}\right) \\
& k_{f}=\frac{k_{f} a_{o}}{a_{o}} \\
& q=\frac{Q_{\mathrm{CO}_{2}} \times \rho}{M_{s f}} \\
& A_{c}=\pi \times \frac{d^{2}}{4} \\
& M_{s f}=M-m \\
& \% m_{\text {inacess }}=\left(\frac{\left(\% m-e_{m o}\right) \times 100}{\% m}\right) \\
& m=\frac{\% m \times M}{100} \\
& \% m=e_{l i m} \\
& k_{f} a_{o}=1,55(U / \varepsilon)^{0,54} \\
& a_{o}=\frac{6 \times(1-\varepsilon)}{D_{p}} \\
& m_{\text {inacess }}=\frac{\% m_{\text {inacess }} \times m}{100}
\end{aligned}
$$


A teoria desse modelo se trata do uso da equação cinética de crescimento microbiano proposto por Monod para representar o rendimento da extração em função do tempo de extração. Foi proposto para descrever curvas de extração globais e por ser um modelo empírico, não considera interações entre o soluto e a matriz sólida e tem apenas dois parâmetros de ajuste [5]. Ele é descrito pela equação abaixo:

$$
e=e_{\lim }\left(\frac{t}{b+t}\right)
$$

Esse modelo necessitou de uma curva experimental para sua utilização, de forma, que com uma curva experimental se fez uma regressão linear e encontrou-se valores relativos a uma equação de primeiro grau que se pode gerar paramentos para a equação do modelo Esquível, como verifica-se abaixo na Figura 3:

Figura 3: Algoritmo para modelo Esquível Fonte: (ALMEIDA, 2006)

$$
\left\{\begin{array} { c } 
{ n a + \sum X b = \sum Y } \\
{ \sum X a + \sum X ^ { 2 } b = \sum X Y }
\end{array} \rightarrow \left\{\begin{array}{c}
a=\frac{\sum Y-\sum X b}{n} \\
b=\frac{\sum X Y-\sum X a}{\sum X^{2}}
\end{array} \rightarrow Y=a+b X \rightarrow\left\{\begin{array}{c}
Y=\frac{1}{e} \\
B=\frac{b}{e_{l i m}} \\
X=\frac{1}{t} \\
A=\frac{1}{e_{\text {lim }}}
\end{array}\right.\right.\right.
$$

\section{RESULTADOS E DISCUSSÃO}

Para modelagem das curvas de extração do óleo de aroeira foram empregados dois modelos matemáticos: o modelo de Sovová (1994) e o modelo de Esquível (1999). Para aplicação dos modelos matemáticos é necessário conhecer algumas variáveis do processo, como densidade do solvente, densidade da partícula da aroeira $(\rho S)$, diâmetro de partícula, solubilidade, porosidade do leito $(\varepsilon)$, altura e raio do extrator obtidos para a condição de 223 bar e $50^{\circ} \mathrm{C}$. As condições experimentais estão contidas na Tabela 1.

Quadro 1: Dados experimentais utilizados para modelagem da curva de extração

\begin{tabular}{|c|c|}
\hline Temperatura & $323,15 \mathrm{~K}$ \\
\hline Pressão & $220,08 \mathrm{~atm}$ \\
\hline $\mathrm{Q} \mathrm{CO}_{2}$ & $23,13 \mathrm{~mL} / \mathrm{s}$ \\
\hline$\varepsilon$ (Porosidade) & 0,3231 \\
\hline $\mathrm{rs}$ & $0,50 \mathrm{~g} / \mathrm{mL}$ \\
\hline $\mathrm{Dp}$ & $0,00168 \mathrm{~m}$ \\
\hline $\mathrm{RCO} 2$ & $0,84 \mathrm{~g} / \mathrm{mL}$ \\
\hline Altura do leito & $0,24400 \mathrm{~m}$ \\
\hline Solubilidade & $3,40 \mathrm{~kg} \mathrm{soluto} / \mathrm{m}^{3}$ solvente \\
\hline Diâmetro do leito & $0,01350 \mathrm{~m}$ \\
\hline Vazão solvente & $1,38775 \mathrm{~L} / \mathrm{min}$ \\
\hline Massa da amostra & $10,00310 \mathrm{~g}$ \\
\hline \% massa do soluto & 3,9804 \\
\hline \% m soluto inacessível & 30,83 \\
\hline Rendimento limite & 3,98 \\
\hline Área transversal & $0,00014 \mathrm{~m}^{2}$ \\
\hline massa mol solvente & $44 \mathrm{~g} / \mathrm{gmol}$ \\
\hline M soluto amostra & $0,39817 \mathrm{~g}$ \\
\hline M amostra livre de soluto & $9,60493 \mathrm{~g}$ \\
\hline
\end{tabular}


A seguir serão apresentadas as curvas de extração obtidas em cada condição de extração, bem como o modelo gerado. O Figura 3 está agrupado a 223 bar e $50^{\circ} \mathrm{C}$.

No Figura 3, pode-se observar que a condição a $50^{\circ} \mathrm{C} / 223$ bar resultou em um bom rendimento experimental na extração realizada. Nessa mesma condição, a curva apresenta exatamente o comportamento descrito por Sovová, em que a parte inicial da curva é linear, passando por uma faixa de transição até estabilizar em um determinado valor.

Figura 4: Gráfico obtido dos dados experimentais $x$ simulado nas condições a pressão de 223 bar e temperaturas de $50^{\circ} \mathrm{C}$ modelo de Sovová.

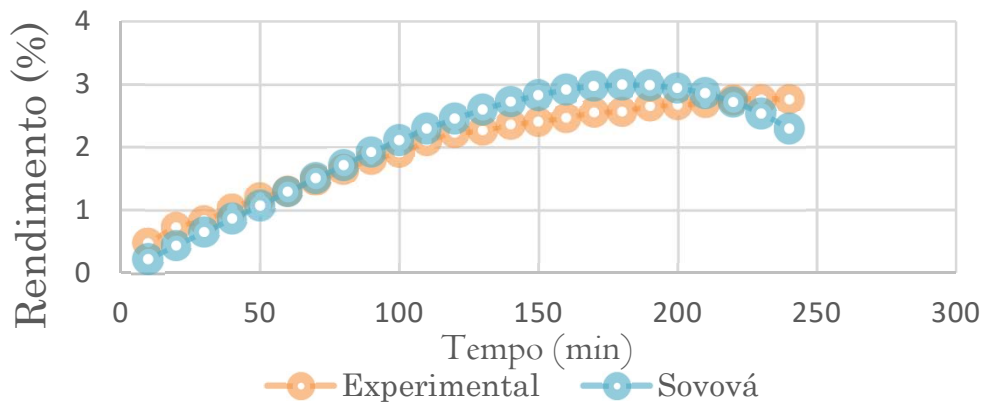

A parte inicial da curva representa uma reta que indica uma taxa de extração de óleo constante, nesta etapa as partículas da matriz sólida se encontram completamente envoltas por uma camada superficial de óleo, nesta etapa a extração é caracterizada pela transferência de massa por convecção entre superfície do sólido e solvente. A etapa de taxa constante de extração segue uma etapa de transição, durante a qual a taxa de extração cai rapidamente. A partir da o processo difusional começa a controlar a transferência de massa, pois o solvente encontra espaço livre para penetração da matriz sólida, solubilização do produto e posterior difusão da mistura óleo -solvente para a superfície da partícula. A última etapa é a taxa quase nula de extração. Nesta etapa a inclinação da curva diminui o traçado se aproxima do valor que representa o conteúdo teórico do óleo extraível de Schinus terebinthifolius Raddi [1].

Além disso, os parâmetros kf, Z, W, Yr e Ks foram gerados para o modelo de Sovová (1994), estes parâmetros foram estimados para as condições anteriores utilizando a técnica numérica que minimiza o quadrado da diferença entre os valores experimentais e calculados pelo modelo. Os coeficientes de transferência de massa da fase sólida e fluida foram calculados a partir destes parâmetros. É possível observar que nessas condições o parâmetro Xk (massa de soluto inacessível dentro das partículas de fase sólida) apresentou os menores valores. Isto significa porque a maior parte do soluto foi extraída pelas duas fases anteriores. No Quadro 2, é possível observar esses os dados referentes aos coeficientes de transferência de massa para pressão de 223 bar e $50^{\circ} \mathrm{C}$.

Quadro 2: Parâmetros estimados e coeficientes de transferência de massa para a pressão de 223 bar e temperatura de $50^{\circ} \mathrm{C}$. 


\begin{tabular}{|c|c|c|c|c|c|c|}
\hline \multirow{2}{*}{$50{ }^{\circ} \mathrm{C}$} & $\mathrm{Xk}$ & $\mathrm{Z}$ & $\mathrm{W}$ & $\mathrm{yr}$ & $\mathrm{kf}(\mathrm{m} / \mathrm{s})$ & $\mathrm{ks}(\mathrm{m} / \mathrm{s})$ \\
\cline { 2 - 7 } & 0,012782 & 1,295483 & 0,058868 & 0,008762 & 0,000441 & 0,000918 \\
\hline
\end{tabular}

kf - coeficiente de transferência de massa de fase fluida

ks - coeficiente de transferência de massa em fase sólida

W - Parâmetro do período de extração lenta

Z - Parâmetro do período de extração rápida

$Y$ - Solubilidade

Xk - Massa de soluto inacessível dentro das partículas de fase sólida

Para o modelo Esquível gerou-se os parâmetros do algoritmo na Figura 4 após a regressão linear que depois foi possível compor o Quadro 3.

Quadro 3: Parâmetros retirados da regressão linear da curva experimental.

\begin{tabular}{|c|c|}
\hline$b$ (calc) & 59,11 \\
\hline $\begin{array}{c}\text { elim } \\
\text { (calc) }\end{array}$ & 3,04 \\
\hline equação & $\mathrm{e}=3,039(\mathrm{t} /(59,1134+\mathrm{t})$ \\
\hline
\end{tabular}

Fonte: Própria (2019).

Figura 4: Gráfico obtido dos dados experimentais $x$ simulados através do Esquível

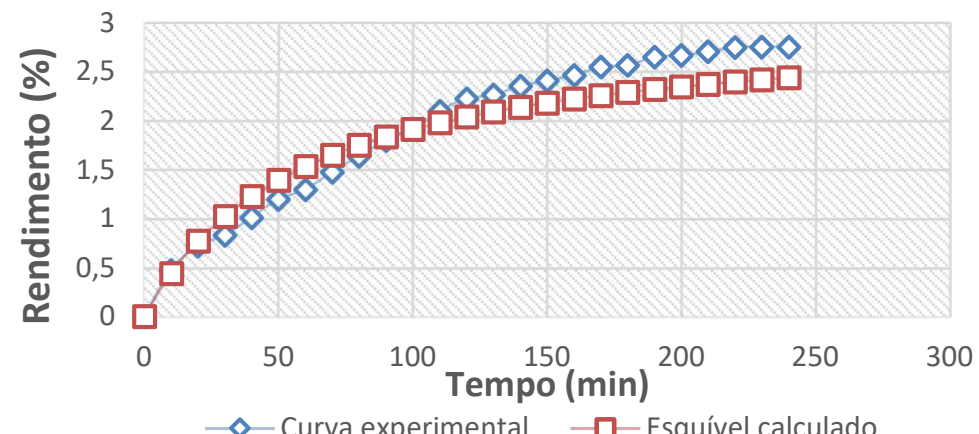

Devido a existência de resíduos no cálculo do modelo os pontos não se correspondem sendo necessário como consequência uma correção com auxílio do Solver@ que levou a novos parâmetros do Quadro 3 e a Figura 5.

Quadro 3: Novos parâmetros.

\begin{tabular}{|l|c|}
\hline$b$ & 104,13 \\
\hline$e(\lim )$ & 3,98 \\
\hline equação & $e=3,9804(t /(104,1301+t)$ \\
\hline
\end{tabular}

Figura 5: Gráfico obtido dos dados experimentais $x$ simulados através do Esquível corrigido

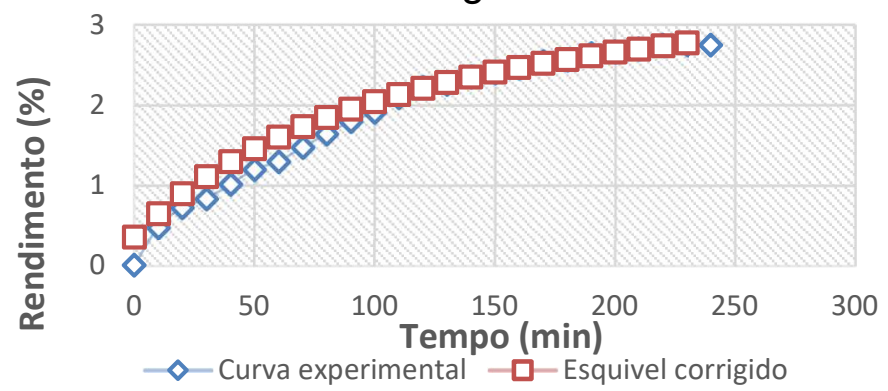


Com o modelo Esquível pode-se observar um comportamente semalhante a Sovova em que a parte inicial da curva representa uma reta que indica uma taxa de extração de óleo constante caracterizada pela transferência de massa por convecção entre superfície do sólido e solvente.

\section{CONCLUSÃO}

O desenvolvimento do presente estudo possibilitou uma análise das curvas de extração do óleo de aroeira com relação aos modelos matemáticos de Esquivel e Sovová. Posteriormente as observações gráficas concluiu-se que o modelo de Sovová possui um rendimento maior que o experimental, como pode-se observar na Figura 4. Após 150 minutos curva de Sovová ultrapassa a curva experimental, por possuir a taxa de extração de óleo mais constante, entretanto os valores não são discrepantes, possuindo uma margem de erro pequena. Já o modelo matemático de Esquivel possui um resultado menos preciso, já que é um modelo mais simplificado utilizando apenas dois parâmetros, negligenciando as dispersões axial e radial.

Ante ao exposto, a curva de extração do óleo da Schinus terebintifolius Raddi mais precisa nas condições de 223 bar e $50^{\circ}$ é a do modelo matemático de Sovová.

\section{Agradecimentos}

Agradecemos primeiramente aos professores Ana Lúcia, Ewerton Emmanuel, Fernando Pellegrini pela oportunidade de fazer parte deste projeto e ao Centro Universitário Senai Cimatec pelo apoio estrutural e tecnológico.

\section{REFERÊNCIAS}

1. ALMEIDA, Priscilla Prates. Extração de óleo essencial de hortelã ( Mentha spicata I.) com misturas de solventes a alta pressão. 2006. Dissertação (Mestrado em Engenharia de Alimentos) - Programa de Pós Graduação em Engenharia de Alimentos, Santa Catarina, 2006.

2. BUSATO, Nathália Viégas et al. Estratégias de modelagem da extração de óleos essenciais por hidrodestilação e destilação a vapor. Ciência Rural, Santa Maria, 2014. Disponível em: http://www.scielo.br/scielo.php?script=sci_arttext\&pid=S0103$84782014000901574 \&$ Ing=pt\&tIng=pt. Acesso em: 19 jun. 2019.

3. H. Sovová. Mathematical model for supercritical fluid extraction of natural products and extraction curve evaluation. The Journal of Supercritical Fluids, Institute of Chemical Process Fundamentals, Academy of Sciences of the Czech Republic, Rozvojová 135, 16502 Prague 6, Czech Republic, p. 3552, 31 mar. 2004.

4. NASCIMENTO, Carolina Menezes. Estudo de Modelos Matemáticos aplicados à cinética de extração de óleos em condições supercríticas. 2017. Monografia (Curso de Graduação em Engenharia Química) FACULDADE DE ENGENHARIA QUÍMICA, Uberlândia, 2017.

5. D, BESSA et al. Modelagem matemática da extração de óleos bioativos de microalgas usando fluido supercrítico. CobeqIC 2015, Unicamp, Campinas, 2015. 